\title{
The Role of Spirituality in the Medical Care of Patients Suffering From Addiction and Mental Disease
}

Rharad Dikra ${ }^{1}$, Benali Abdeslam ${ }^{2 *}$

${ }^{1,2}$ Department of Psychiatric, Military Hospital “Avicenne”, Marrakech, Morocco

\author{
"Correspondence author \\ Benali Abdeslam \\ M.D, Ph.D \\ Departement of Psychiatry \\ Faculty of Medicine and Pharmacy of Marrakesh \\ University of Cadi Ayyad \\ Morocco
}

Submitted : 20 Jun 2020 ; Published : 5 July 2020

\begin{abstract}
For a long time, a separation has been made between medicine and spirituality, but since the 90s, there has been an advent of spirituality after several researchers in North America have found that this dimension is linked to several pathologies and could play a role in the recovery of psychiatric patients. After a brief reminder about spirituality and religion and some concepts of mental health, we present three qualitative studies. The first aims to measure the degree of religiosity of the consultants of the center of addiction of Marrakech by using A religiosity Scale created by DR Dany R.Khalaf. The result of this first study is unequivocal, the patients of the center of addictology have a middle degree of religiosity with a certain profile a clear male predominance having less than 30 years, sedentary. Half of them is unemployed and did not passed secondary school in non-religious schools. Mostly Muslim, while occasionally consuming alcohol and consuming more than 10 cigarettes a day.
\end{abstract}

The second study attempts to establish a link between religiosity and the impact it may have on the abstinence of alcohol addicts. The final result of this study is that religiosity has a definite impact on alcohol dependent abstinence and is even the main reason behind the abstinence of some patients.

The third study sought to demonstrate the relationship between religion and suicidality among depressed patients. The result is that religion is one of the reasons for abandoning suicidal ideation and that it brings tremendous benefit to patients, which make them quit the idea of committing suicide. During both studies, questions relating to the integration of the spiritual and religious aspects of care were asked. The majority of patients want spirituality and religiosity / religion to be taken into consideration and integrated into their care while being with their doctor. After having seen all the benefits of spirituality and the positive effect it had on patients in the various studies, we can only conclude that spirituality has a primordial and very important role in the care of patients. . Herein, everything needs to be done, a protocol of care taking should be established, including this dimension and future psychiatrists needs to be trained to do it, and it should include a panelist such as "the leaders of the cults" in the care.

\section{Keywords : Spirituality, Addiction, Mental Disease, Medical Care}

\section{Introduction}

Since the emergence of modern medicine, there has been a clear separation between spirituality / religiosity and medicine. Only one model has emerged as the dominant paradigm in medical management, it is based on the establishment of diagnosis, identifying the disorder or disease, researching its cause and initiating the treatment to eliminate this cause. This model is certainly very effective when it comes to treat an infectious

disease; however its effectiveness remains limited when the cause of the disease is social, environmental, psychological or behavioral.

But since the 1990s, an improvement has been noted, researchers in psychiatry are interested by the spiritual side and has already admitted that it has some impact on mental health. In addition, 
the WHO has defined "spirituality" as: "The aspects of human life related to experiences that transcend sensory phenomena are called" spiritual ". It is not the same thing as the - religious - although for many people the spiritual dimension of their life contains a religious element [1].

Also while talking about religiosity we can say, that it is understood as the set of practices lived by a person within some religion. Although its meaning has evolved, at the present time religiosity may be defined as sentimental attraction to religion and divinity or to both. In our society religiosity is an integral part of spirituality. To explore this spiritual side we conducted three studies:

- The first concerns the evaluation of the level of religiosity of the patients of the center of addictology/rehab center of Marrakech using the religiosity scale of Dr. Dany R.Khalaf

- The second attempt to demonstrate the impact of religiosity on the abstinence of alcoholics

- The third seeks to establish the link between suicidality and religion in depressed patients

\section{Patients and Methods}

Study 1: Evaluation of the religiosity level of the patients We conducted a descriptive study on 30 patients of the Addictology Center in Marrakech, to whom we inoculated the "religiosity scale" developed by Dr. Dany R.Khalaf [2]. We included all patients over 18 years old and excluded all patients suffering from dementia, psychosis and illiterate people. The agreement of the patients was taken and the questions related to the investigation were asked only after the end of the consultation and after verifying that the patient had his prescription, in order to avoid any subjective fear from the patient and to avoid him thinking that his medical care may be altered by his refusal to participate in the study. After, all data was collected, a descriptive analysis was made using the google froms software.

Study 2: religiosity and its impact on abstinence in alcohol addicts

We conducted a cross-sectional qualitative study in the addictology center of Marrakech, we included 10 patients suffering from alcohol addiction already in abstinence or having experienced a period of abstinence. We included patients older than 18 years and we excluded the patients suffering from psychosis, dementia or illiterate people. After responding to the "religiosity scale" of study number 1 , they participated in a semi structured interview in face to face, during the interview they answered more than 16 open or closed questions about religiosity, spirituality, abstinence and alcohol addiction/abuse. The data were collected using a mobile phone and the interviews were transcribed literally in Darija or in French according to the patient's choice using the conventional rules of punctuation and stage directions to make the non-verbal readable.

Data processing was done through thematic analysis and using the MAXQDA2018 software. The analysis was obtained through several stages: the impregnation, establishing themes, elaborating a grid / table of analysis and finally coding and an analyzing.

\section{Study 3: Suicidal behavior and religion among depressive} patients

We conducted a cross sectional qualitative study at the addictology center in Marrakech. We included 6 patients over 18 years old, followed up at the center and suffering from depression and we excluded patients under 18, patients suffering from psychosis or dementia and illiterate people. The data collection was made after inoculating the "religiosity scale" of Study 1, after this patients participated in a semi structured face-to-face interview, in which they answered more than 17 open and closed questions and. The data collection was done using a mobile phone followed by a word-by-word transcription of the interviews written in Darija using the conventional rules of punctuation and stage directions to make the non-verbal readable.

Data processing was done through thematic analysis and using the MAXQDA2018 software. The analysis was made through several stages: the impregnation, creating themes, elaborating a grid / chart of analysis and finally coding and and analyzing.

\section{Results \\ Result of Study 1:}

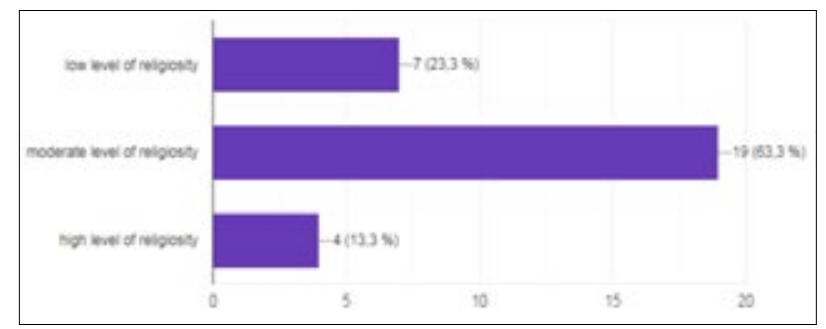

Illustration 1: level of religiosity

More than half of the patients $63.3 \%$ had a moderate level of religiosity, followed by those with a low degree of religiosity $23.3 \%$ and only $13.3 \%$ had a high level of religiosity

\subsection{Sociodemographic characteristics}

a) Age

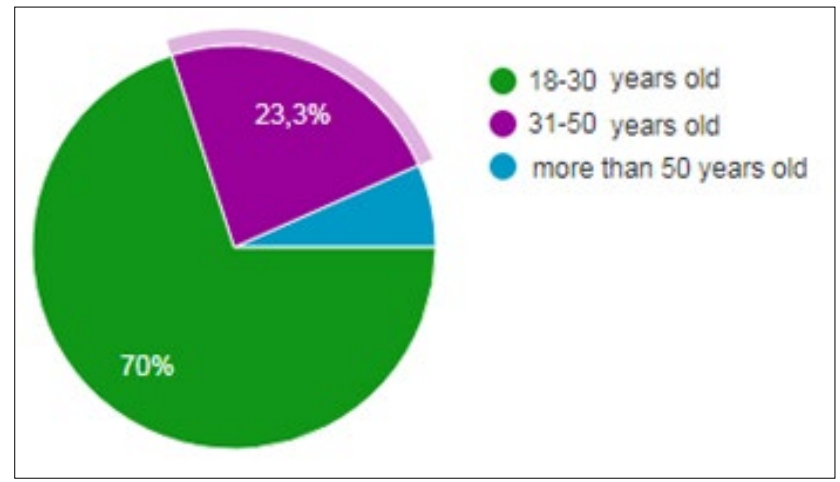

Illustration 2: age of the patients 
Most patients are between 18 to 30 years old, followed by the 31 to 50 years who represented $23 \%$ while the over 50 years are a minority only $6.7 \%$.

\section{b) Gender}

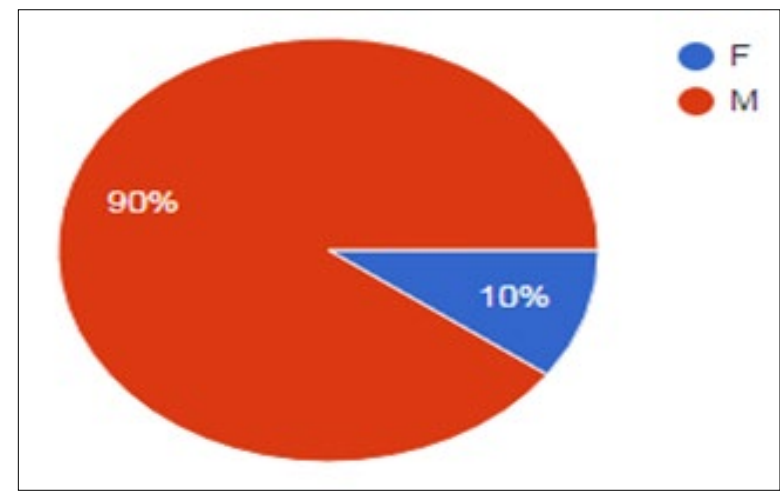

Illustration 3: Gender of the patients

More than three quarters of the study population are men (90\%)

c) Marital status:

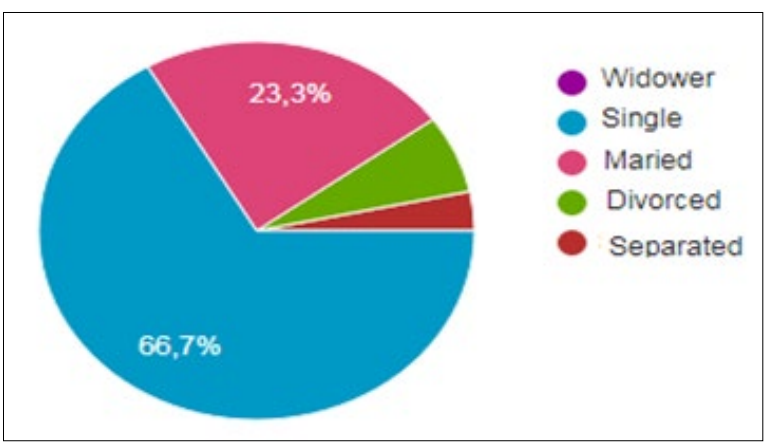

Illustration 4: Marital status of the patients

More than half of the population is single (66.7\%), followed by the married people at $23.3 \%$, divorced or separated persons are few

\section{d) Professional situation}

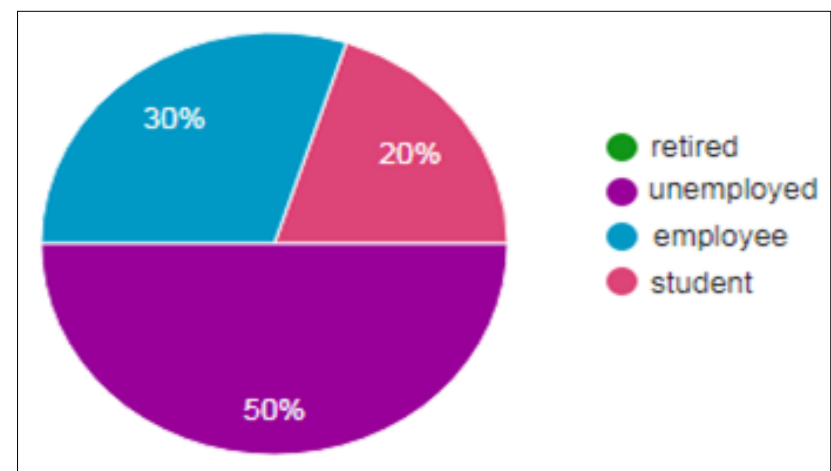

Illustration 5: professional situation of the patients

Half of the population is unemployed $(50 \%)$, the other half is either working $(30 \%)$ or studying $(20 \%)$.

\section{e) Educational level}

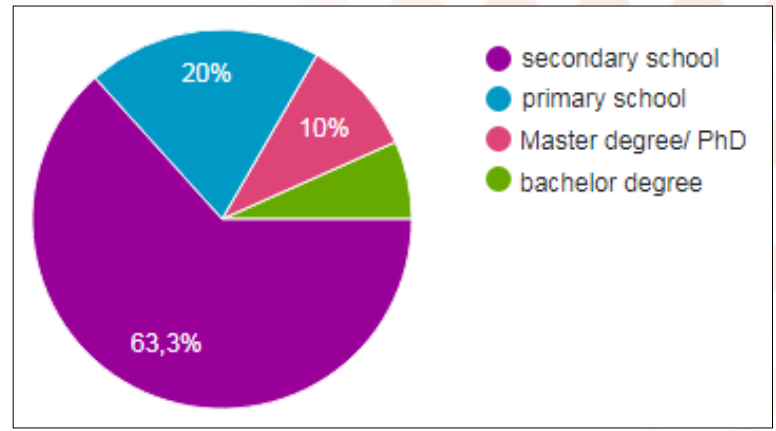

Illustration 6: educational level of the patients

Many patients stopped schooling in high school (63.3\%), 20\% of them did not go beyond primary school while only $10 \%$ have a master's or $\mathrm{PhD}$

\section{f) Type of schools attended to}

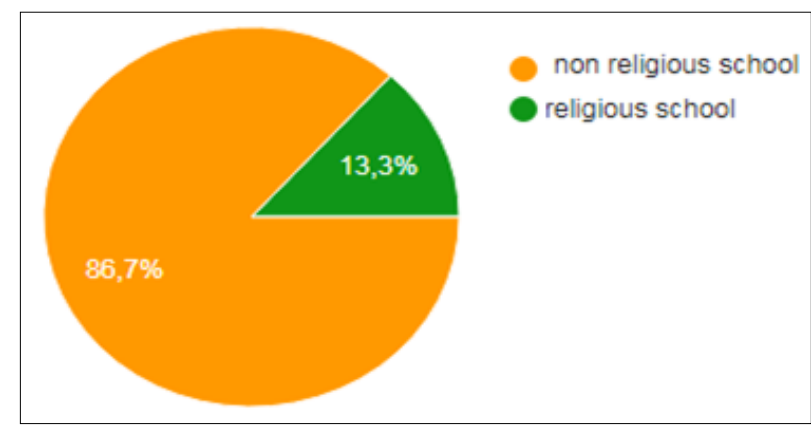

Illustration 7: Type of schools attended to by the patients

The vast majority of patients completed most of their schooling in non-religious schools (86.7\%) compared to only $13.3 \%$ who spent most of their schooling there

\section{g) The practice of sport}

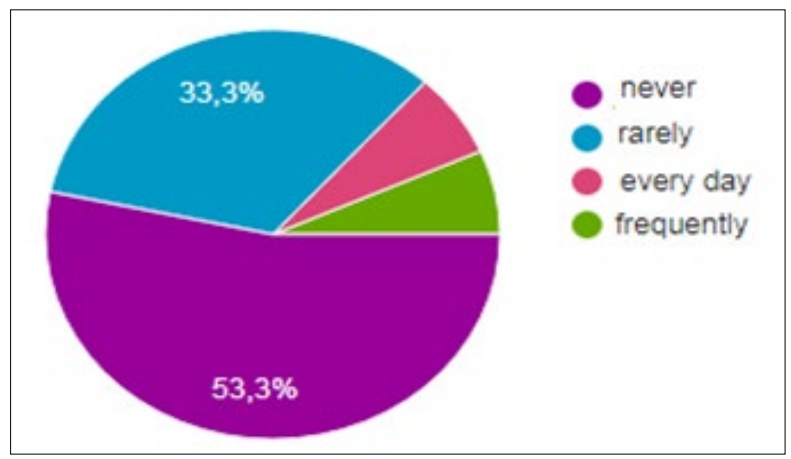

Illustration 8: practice of sports by the patients

More than half of patients do not practice sports $(53.3 \%)$, followed by $33.3 \%$ of patients who rarely do so. 
h) Cigarette consumption

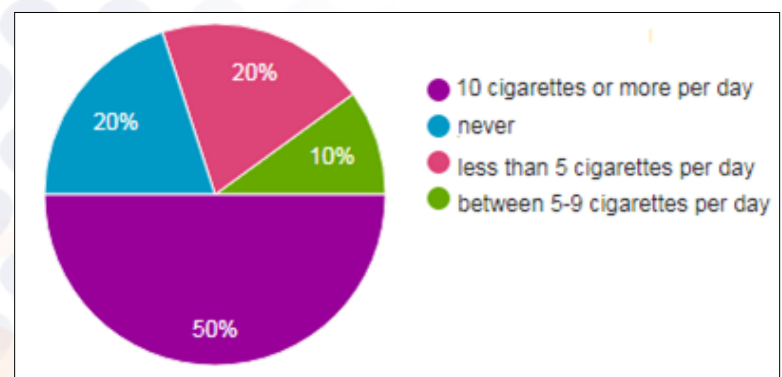

Illustration 9: Cigarette consumption

Half of the population smokes more than 10 cigarettes a day, followed by those who never consume cigarettes $(20 \%)$ and those who consume less than 5 cigarettes a day (20\%), only $10 \%$ of the population consume 5 to 9 cigarettes a day

\section{i) Alcohol use}

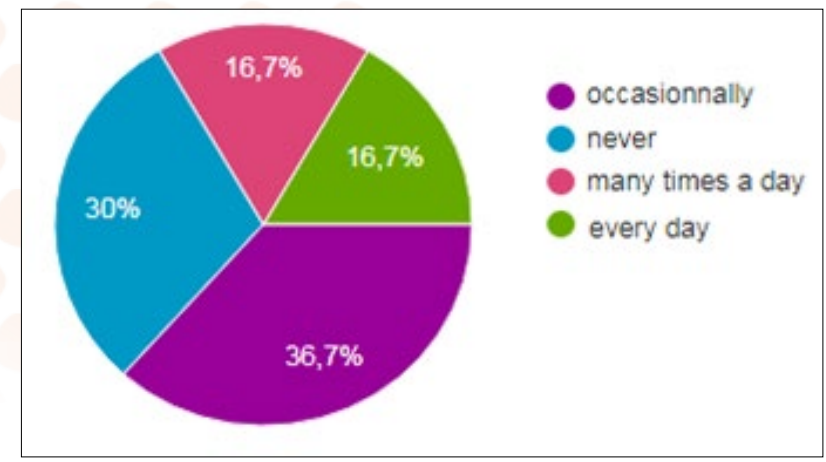

Illustration 10: alcohol use
Just over one-third (36.7\%) of the population consume alcohol occasionally, followed closely by $30 \%$ who never consume alcohol, the rest of the population consume equally each day $(16.7 \%)$ or several times in the same day (16.7\%).

\section{j) Religion}

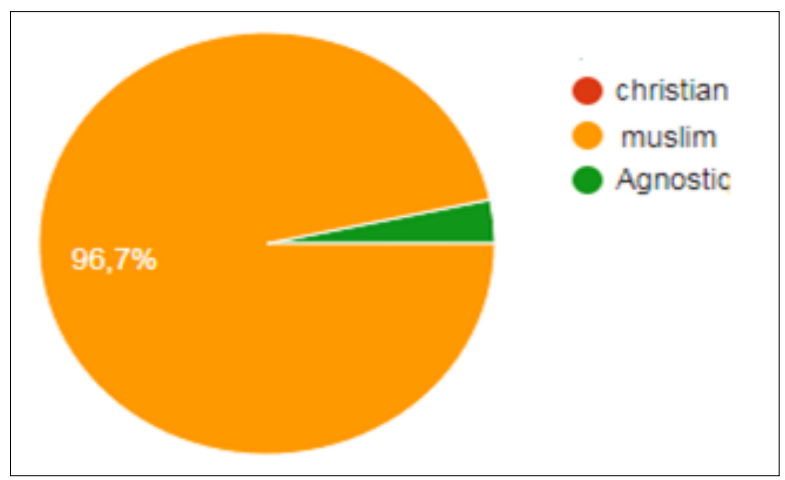

Illustration 11: Religion of the patients

Most of the patients are Muslims 96,7\%

\section{Resullt of study 2:}

To make things reader easier here are the most important results

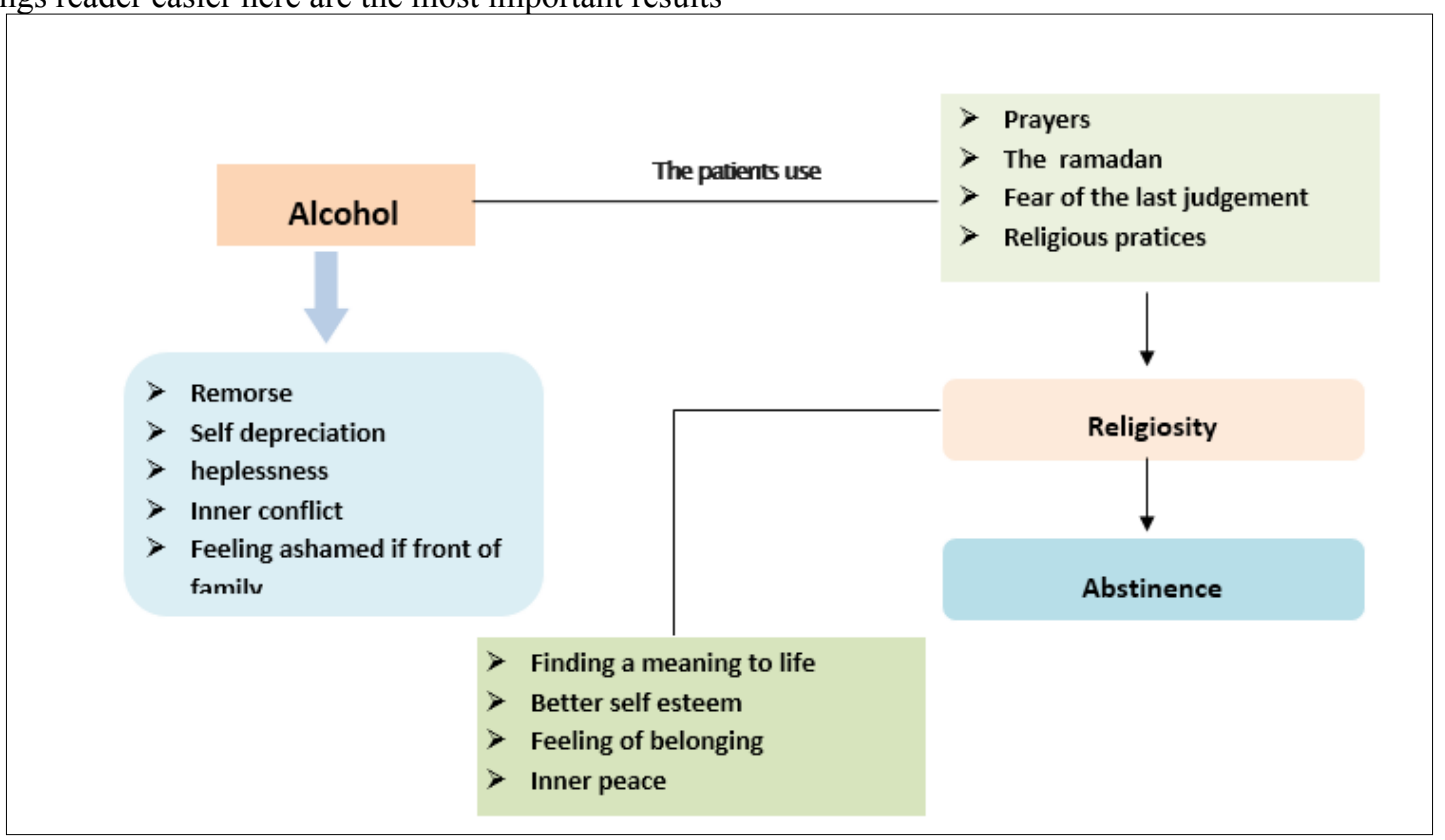

We arrived at the result that patients suffering from alcohol addiction experience remorse, suffer from self depreciation, feel helpless about consuming alcohol and ashamed in front of their family, but they had resorted to prayer, Ramadan, but also the existence of the permanent fear of the last judgment in order to abstain from consuming alcohol. 
These practices and these reasons themselves are part of the religion and they led them towards abstinence while helping them finding a meaning to their life, a better self-esteem and inner peace.

We should also notice that during the study it turned out that the religion is "everything" for the patients, they even idealize the religious person and many consider themselves as half religious or just believers.

Result of study 3:

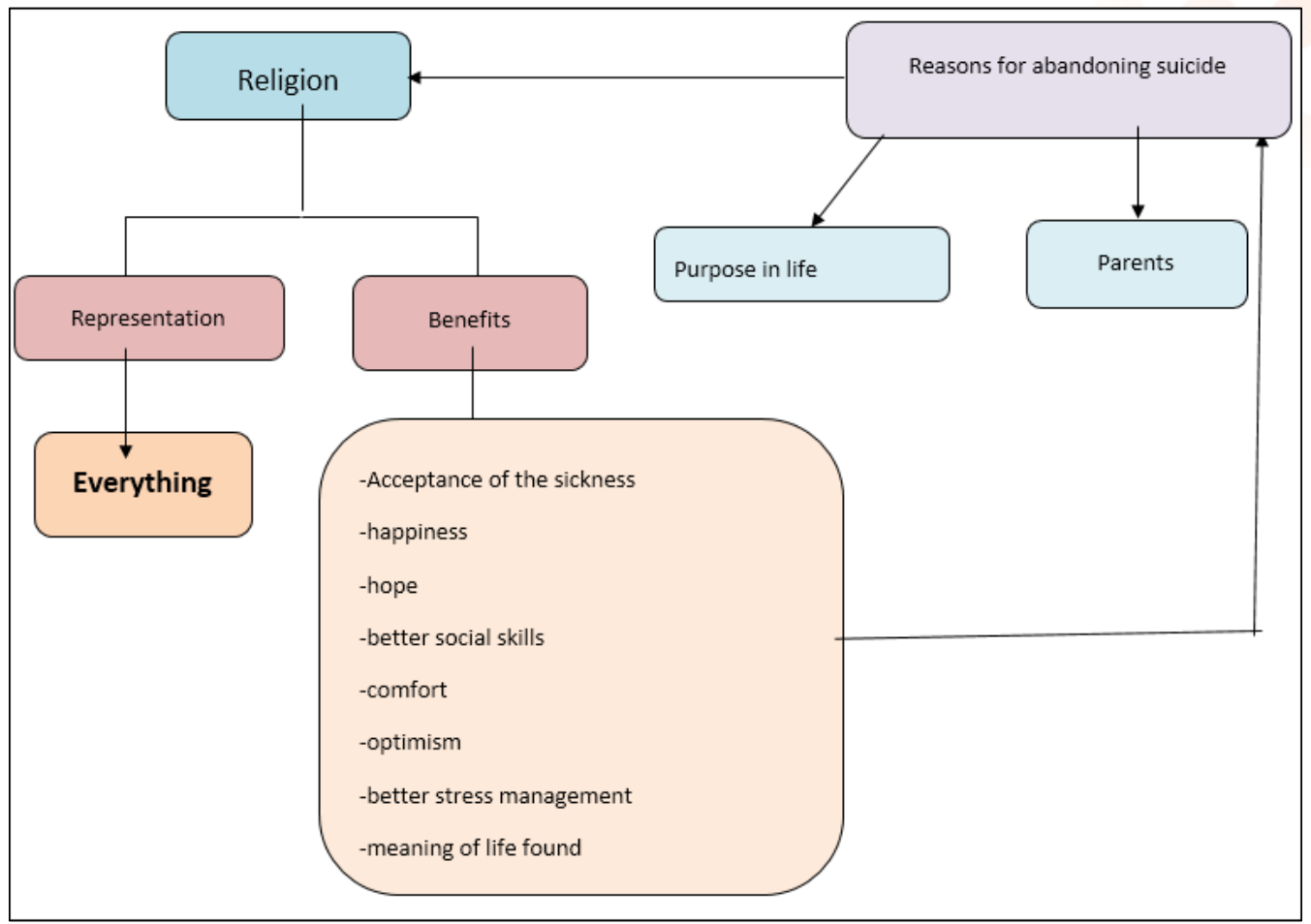

Among the reasons why our depressive patients abandoned suicidal acts and ideas, we find the religion which they consider as "everything" in there lives and which helps them to accept their disease, gives them happiness, hope, comfort, optimism, allows them to have a better stress management technics and helps them to find the meaning of their life. It is obvious that when we acquire all this we won't commit suicide.

\section{Discussion}

In the study number 1 , we found out that most of the patients have a moderate level of religiosity,but while we do researches about religiosity and consumption of substances, in the literature we find two relevant studies which tried to link the religiosity / religion and the consumption of drugs : A first study was conducted in Canada by Edward M. Adlaf and Reginald G. Smart on participants (Protestants, Catholics, without religious affiliations). They found that Catholic students were less drug users compared to Protestants or those without religious affiliation and this is for cannabis, other drugs, or hallucinogenic drugs. They came to the conclusion that religiosity and church attendance are strongly related to drug use. Those who consider themselves very religious were the least alcohol and other drug users: $61 \%$ versus $76 \%$ for alcohol, $8 \%$ versus $29 \%$ for cannabis, $6 \%$ versus $23 \%$ for drug prescriptions (not drugs), $10 \%$ versus $18 \%$ for drugs and $3 \%$ versus $17 \%$ for hallucinogenic drugs [3].

A second study was conducted by Khalil Akhtar Khavari on both Christian, Jewish, Catholic patients and other religious affiliations, the study involved 4853 people and found a strong relationship between the level of religiosity and consumption. In general, patients seeing themselves as "very religious" consumed less alcohol and psychoactive substances compared to those who considered themselves "non-religious". Much higher consumption of tobacco, cannabis, amphetamines and alcohol was found among non-religious groups [4].

Our Muslim patients have a moderate degree of religiosity for $63.3 \%$ and low level of religiosity for $23.3 \%$ this partly explains they suffer from addiction, and why for many of them the consumption of alcohol is occasional and why half of them smoke more than 10 cigarettes a day. Regarding substance use and religious affiliation, a study was conducted by ACHEAMPONG YAW AMOATENG and STEPHEN J. BAHR at Brigham Young University on 17,000 students to demonstrate the relationship between religion and drug use. They found that religious affiliation is related to drug use: Mormons were the least drug-consuming group. Baptists and fundamentalists also had a low rate. Those with no religious affiliation were the biggest cannabis users [5]. 
Thanks to this latest study, we can say that populations with a high level of religiosity are less likely to end up suffering from addiction. Religion provides a type of "moral code" in which religious people believe and which implies the immorality of drug use no matter what's the religion. Our patients having a moderate level of religiosity, it makes sense that they suffer from addiction.

In the study number 2 , the participants in the study do not consider themselves religious, they see themselves as believers or as half religious. When we look at the literature on the religiosity and addiction to alcohol among Muslims,we only find two other studies were done on Muslim populations to demonstrate the relationship between religiosity and alcohol addiction (the one in Turkey and the other in Lebanon): The 2008 Ozbay Turkish study of 974 students at "Nidge University" found that only $34 \%$ of students were drinking and that consumption was significantly lower among those discussing religion with their family and close friends [6].

The Lebanese study, conducted by Ghandour and colleagues on 1837 students, both Muslim and Christian, indicated that only $43.8 \%$ of Muslims had a history of alcohol consumption compared to $87.5 \%$ of Christians. And Muslim students engaged in religious practices suffered much less from alcohol addiction [7].

This joins the results of our study, our patients considering themselves as half religious therefore are not being very committed to religious practice, this explains why they are alcohol addicts. One hypothesis could explain the perfect sync of our results with other studies, the religious practices requires a strong committement and a diligent practice every day, something which requires that the person stays sober, so the more he will practice the less he will consume.

Many of our patients mentioned the fact that religious reasons and practices were behind their abstinence, and therefore that religiosity was responsible for their abstinence.

Related to this subject of religiosity and abstinence, in literature we find two studies: one made in 2007, based on the US National Alcohol Survey, confirmed that religiosity was strongly associated with alcohol abstinence [8].

The other prospective study conducted by Trond Nordfjærn from 2002 to 2008 , conducted in subjects over 40 years of age in Norway, showed that religiosity was strongly associated with abstinence but also with a decrease in consumption [9].

These studies only confirm our results. It is obvious that if a person wishes to practice his religion according to the precepts, he must become abstinent to be able to ensure the various actions that are required from him.

Finally, it should be noted that during our study, it turned out that for the patients the religiosity was essential and important, the religion means "everything" for them even though they did not consider themselves as really religious or see themselves just as half religious. This explains their old addiction. Yet it is for religious reasons and through religious practices (prayer, fear of last judgment, Ramadan, and other religious practices) that they manage to stay sober.

As studies have shown it before, religiosity is strongly associated with abstinence. Thus we can say that religiosity has a significant impact on the abstinence of alcohol addicts being even behind the abstinence of some patients.

This is very much in line with the results of the various studies on religiosity and abstinence.

\section{The strengths of the study:}

All the studies that have been done to establish a link between religiosity and abstinence have only confirmed the close link between the two. However, none of them explained how religiosity influenced abstinence or the effects of religiosity on patients, this is the contribution of this study.

In the study 3, Our depressive patients, although they consider religion as "everything", after a careful reflection on the definition of religion and how they do much the definition they have given, they tend to see themselves as believers without much practice and one of them does not have religious affiliation.

Michael E McCullough and David B Larson conducted a review of the literature, consisting of about 80 studies on the relationship between religion / religiosity and depression. They concluded that some people with no religious affiliation were at greater risk of developing depression compared to those who did have one. While people with a high level of religious involvement and for whom religion is of great importance have a low risk of developing depression [10].

This review corroborates our study, since our patients were not very religiously involved this explains why they are suffering from depression. In our study, the patients did not consider themselves religious and half of the participants already had suicidal thoughts but only one had already done a suicide attempt.

In the literature, we find an analysis made by Pritchard and Amanullah in 2007, according to data from the WHO to compare the number of suicide in England with those of 17 Muslim countries. Countries were then ranked by the level of religious practice, ranging from the lowest countries (Azerbaijan, Kazakhstan, Kyrgyzstan, Tajikistan, Turkmenistan, Uzbekistan) to those with the highest level of religiosity (Middle Eastern countries of Bahrain, Egypt, Kuwait, Iran, Qatar, Syria) .The researchers found that the highest rates of suicide were in the least religious countries and that the lowest were in the most religious countries [11].

The patients in our study are not religious, this explains why half of them have suicidal thoughts, while the other half who 
do not think about suicide, they also said that the "religious ban" around suicide was among the reasons that prevented from thinking about it, one study came to the same conclusions and the same reason. Eskin's 2004 study of 420 students in Turkey attempted to demonstrate the link between suicide and religion: Two groups of students were made the first were educated in a religious school and others in a secular school. The results showed that students in secular schools had more suicidal ideation than those with religious education because they think that those who commit suicide would be punished after death [12].

Also, half of our patients do not have suicidal ideation, but unanimously consider religion as very important and essential. In the literature, another study by Zuraida and Ahmad in Kuala Lumpur examined the relationship between suicidal ideation and depression. The study of patients with depression or depression in bipolar disorder demonstrated that patients for whom religion was important and the main source of life had significantly fewer suicidal thoughts [13].

A hypothesis seems quite interesting to us and could explain all the previous results, during our development we determine a "vision of the world" of our own, which is also influenced by the religious context in which we evolve. This "worldview" concerns the reasons for our presence on earth and the fate that will be reserved to us after our death, religion provides us with a "material" to guide us in our beliefs on the subject. Suicide is an act if "succeeded" leads to death. We can quite legitimately think that what our "worldview" suggests about the results of this act, will necessarily have a great influence on our determination to accomplish it. It is indeed difficult to doubt that an approach leading to death will be considered differently depending on whether we believe that there is simply nothing after or that we believe that dying will lead us to an idyllic heaven or to hell. Among the contributions of religion to our patient's life, we find happiness that is what a study tried to prove. A study conducted by Abdel-Khalek and Eid on more than 1937 students in Kuwait and 1009 Palestinian students examined while trying to establish the link between religiosity / religion, well-being and depression. At the end of the study it turned out that religiosity was positively related to happiness and well-being and inversely related to depression [14].

One of the benefits that a patient needs is this one, religion brings optimism to our patients. To be able to affirm this, we find a study made in Algeria which examined the relationship between religiosity and optimism in more than 2000 participants, half of whom were between 18 and 25 years old, to reach the conclusion that religious practice was linked high level of optimism [15].

During our study, we saw that religion brought many things to patients: acceptance of illness, happiness, hope, comfort, optimism and a meaning of life refounded again and all of this happened without involving religion in the medical care of the patient but it was used by the patient himself. While the majority of patients think that we must integrate the religious and spiritual side in their medical care and think that it will help them.

The initiative has already been taken in other countries. In the literature, we find that three randomized clinical trials were done on Muslim depressive in Malaysia, the first two by Azhar and Varma:

- The first involved two groups: one group had benefited from conventional therapy with antidepressants, the other group had also received an "Islamic psychotherapy", an Islamic psychotherapy, patients were followed over the first 3 months and those who had benefited from Islamic psychotherapy were improving faster [16].

- The second, included 30 patients who had experienced bereavement, one group benefited from conventional psychotherapy and antidepressant treatment and the second was entitled to "The additional religious psychotherapy" involving the discussion of issues and religious themes relevant and important to the patients. The second group after 6 months showed a significant improvement [17].

- The third by Razali, included 203 patients divided into two groups: the first received a conventional therapy as for the other group he benefited from a conventional psychotherapy in addition to a religious psychotherapy (religious psychotherapy), using cognitive techniques guided by quoran and hadith. Both groups were followed over 6 months, the group receiving religious psychotherapy responded much more quickly than others to treatment [18]. These three clinical trials confirm that the integration of religiosity in the care of patients suffering from depression can only help them to get better, as our patients, who had used it on their own to "get better" given the lack of this approach in their care.

\section{The strengths of the study:}

All the studies done before, have revealed that religiosity / religion has a positive influence on depression, but none has demonstrated the contribution of this religiosity on patients, since all these acquired the patient cannot rethink about committing suicide. Just as studies have confirmed that religiosity decreases suicide and suicidal ideation, very few have considered the reason for this. And it is in these points that lies the strength of our study.

In summary, In the study we conducted, the level of religiosity of the patients was moderate which explains that they suffered alcohol addiction.

Yet the following study also showed that religiosity (through some practices) led to abstinence.

Very few studies have examined the link between acohol dependence and religiosity / spirituality in Muslim populations, only $1 \%$ of published studies concern these populations [19].

But in his book "health and well being in Islamic societies," Dr. Harold G. Koenig after comparing several studies linking religiosity and alcohol addiction came to the conclusion that 
a significant level of religiosity / religious involvement is linked to a lower alcohol dependence rate. And that alcohol dependence is generally less important among Muslims than among other religious groups [20].

Reading the conclusion of this latest study, we cannot deny the fundamental role of religion and we can only ask that there is more research on this side so that the Muslim religion is used to problem of alcohol dependence. It should be noted that more than three quarters of the participants in study number 2 , would like the spiritual and religious dimension to be included in their care, we will evoke one of the most interesting examples in modern history on the use of spirituality / religiosity among alcoholics addicts, it is the program of alcoholics Anonymous (AA) [21] whose program of 12 steps has a religious / spiritual dimension in its Anglo-Saxon form and this is even palpable in its French version.

At the end of each meeting, members of the Alcoholics Anonymous group stand between them and quote a prayer, called the Serenity Prayer: "My God, give me the serenity to accept the things I cannot change, the courage to change the things I can, and the wisdom to know the difference [22].

Participation in alcoholics anonymous has been shown to be more effective in maintaining abstinence compared to other self-help groups in studies in the United States [23].

Study participants have a moderate degree of religiosity / spirituality, which explains why they suffer from depression and that half of them have suicidal thoughts.

Yet for many religion (religious prohibition) is behind their abandonment of suicidal ideation.

Religion has brought a lot to these patients:

- Hope and optimism, which is also reported 12 out of 15 studies investigating the link between religiosity and optimism or hope [24]. Religious beliefs tend to improve optimism and foster positive thinking.

- the meaning of life found, 15/16 studies on the subject have arrived at the same conclusion. This would be due to the fact that religion gives meaning and purpose to life. Religious teachings make us learn that existence is not the result of chance but the work of a creator.

One hypothesis can explain our results: spiritual and religious beliefs encourage positive feelings and values such as love, honesty, joy, peace, hope, patience, generosity, forgiveness, kindness, kindness, compassion ... All these values can only be beneficial and have positive effects on mental health and social interactions. However a spirituality that would be positive, could reduce the lack of self-esteem and hope that can accompany psychic suffering. It can also reduce stress and make sense of life. Spiritual practices can reduce the isolation of the patient (collective prayers, religious classes ...).

A majority of the patients participating in study number 3 said they wanted the spiritual and religious side to be integrated into their care and thought that it would help them, the existence of this help was well proven during the studies and the clinical trials done on Muslim populations with the same pathology. This help is obtained through the effect of religion / religiosity on the patient, which in the literature has already been proven since religious practices and beliefs are constantly linked to feelings of satisfaction, happiness, and positive thoughts and to be well [24].

That's what a depressed patient needs and that's what needs strengthening in depressed patients to help them get out of it. Of course this will only be done by integrating the spiritual / religious aspect in the care of the patients.

\section{Conclusion and Perspective}

Before the 20th century spirituality and science were seen as incompatible, fortunately towards the end of the 20th century, researchers found that spiritual / religious beliefs and practices are widespread in the American population and that they might have clinical relevance. As a result, many organizations of health professionals have emerged, raising awareness of the role of spirituality and religion in the care of patients such as "The American Psychiatric Association", "The American Psychological Association", "The Association of American medical Colleges $\gg . .$.

The concept of health has, since the last few decades, expanded far beyond the notion of the absence of biological disease. Mental health is strongly influenced by the individual's culture, personal relationships and the quality of his social relationships, social context, also his philosophical ideas including the meaning that he gives to his life. Many people with psychological, emotional and stressful life events take refuge in religion to find comfort, hope and meaning in their lives. This shelter can help a certain amount of people; it is to confirm that this study was made.

\section{Spirituality / religiosity and addiction:}

Religion promotes a healthy lifestyle and provides a "moral code" in which substance use/abuse is prohibited and generally health-damaging habits such as smoking and drug use are deterred in religious teachings.

In our studies we have found that religiosity gives strength and reason to some people to resist temptations and to maintain the abstinence of addictive substances, but would also ensure a better self esteem.

Religious practices provided inner peace to withstand stressful life events and life difficulties, thus reducing the need to use drugs to manage stress. Religion would also help to find a sense of one's life and events.

\section{Spirituality / religiosity and depression:}

Religion / religiosity/spirituality would be a great help since it's providing a source of comfort. It promotes the acceptance of his illness also gives hope to the patient, helps to be optimistic and serene in thinking about the future because the patient has already found the meaning of his existence, it gives him 
a better stress management skills and better social relations.

Also according to what was previously seen in reviews about depression, religiosity reinforces resistance, which is the ability to recover and recover more quickly.

\section{Spirituality / religiosity and suicide:}

It is known suicide is prohibited in all major religions.

As we saw in our study, religious investment is negatively associated with suicidal behavior. Spirituality and religion interact with psychological factors such as self-esteem, acceptance of illness, and social factors such as social relationships. When suicidal thoughts are numerous, spirituality seems to be protective and help preventing suicidal attempts. It also appears as an important resource to cope with life's hardships, helps to give hope and promote optimism. It also helps finding a meaning to patient's life which loss is essential in suicidal attempts.

The spiritual evaluation of people with suicidal behaviors seems to be imperative in order to know the representation they have of their role on earth and thus the reasons and meanings why they should stay alive.

\section{The role of spirituality in clinical practice:}

Every human being on earth wants to live in perfect shape, with the minimum of psychological suffering, while having an inner peace and finding a meaning to his life, he also wants to be satisfied with his existence, even if he is a psychiatric patients.

If health professionals continue on neglecting the spiritual/ religious aspect of the human being while focusing only on the biological nature of diseases, they may miss out the critical needs of their patients. Helping a patient, especially in psychiatry and addictology, consists on allowing him to have a better psychological coping, to enrich his positive experiences, to help him find a meaning to his life and to be satisfied with it.

During our studies, we realized the importance of spirituality / religiosity for the patients as well as their desire that this dimension must be taken into consideration by their doctor while healing them; they do also have a firm conviction that this kind of approach is going to help them.

The different results of our studies allow us to think that if we consider the spiritual dimension/side as part of the individual sphere of our patients, this would imply ignoring an important resource that would help them face different difficulties of life, and help us to treat addictions .... Due to this, it is important to continue to explore the peculiarities of spirituality and religiosity in the Moroccan Muslim context. This would allow a better understanding of the spiritual dimension, its evolution according to age and to see more clearly the role that could spirituality play in the global wellbeing and its importance as a coping resource to face the disease.

\section{Perspectives}

Clinicians should inquire about the religious and spiritual activities of their patients, appreciate and encourage these resources if it allows a better psychological and social functioning.

Physicians are rarely trained to deal with the spiritual and religious aspects of their patients. So addressing a patient to a representative of the patient's religion is sometimes problematic. Indeed, many representatives of religions lack psychiatric expertise and are unable to define the boundary between what is spiritual and what belongs in the medical field.

How can we solve this dilemma? Three approaches are possible:

- The first is to help religious institutions develop their scientific knowledge and and train them on psychological counseling.

- The second approach would be to help physicians develop skills to a better understanding of spiritual issues and to define the boundaries between what is medical and what is spiritual.

- A third possibility would be the coordination and collaboration between health professionals and spiritual representatives in the form of workshops that they would lead together.

\section{References}

1. WHO; Treatment of cancer pain and palliative care "; in: Report 804 - chapter $7: 7.1$

2. Dany R. Khalaf, Sani A.A. Hlais, Ramzi S. Haddad, Chantal M. Mansour,Antoine J. Pelissolo, Wadih J. Naja (2014) Developing and testing an original Arabic religiosity scale Middle East Current Psychiatry 21: $127-$ 138.

3. Edward M. Adlaf, Reginald G (1985) Smart Drug Use and Religious Affiliation, Feelings and Behaviour British Journal ofAddictim 80: 163-171

4. Khalil Akhtar Khavari, Teresa McCray Harmon (1982) The Relationship between the Degree of Professed Religious Belief and Use of Drugs The International Journal of the Addictions 17(5): 847-857.

5. ACHEAMPONG YAW AMOATENG STEPHEN J. BAHR (1986) Brigham Young University- RELIGION, FAMILY, AND ADOLESCENT DRUG USE Sociological Perspectives 29(1): 53-76

6. Harold H.G Koenigsaad al shohaib (2014) Health and wellbeing in Islamic societies (alcohol use/abuse) p155.

7. Ghandour LA, Karam EG, Maalouf WE (2009) Lifetime alcohol use, abuse and dependence among university students in Lebanon: exploring the role of religiosity in different religious faiths. Addiction 104(6): 940-948.

8. Laurence Michalak, Karen Trocki, Jason Bon (2007) Religion and alcohol in the U.S. National Alcohol Survey: How important is religion for abstention and drinking? Drug and Alcohol Dependence 87: 268-280.

9. Trond Nordfjærn (2018) Religiosity and Alcohol Use: Is Religiosity Important for Abstention and Consumption Levels in the Second Half of Life?, Substance Use \& 
Misuse.

10. Michael E McCullough, David B Larson (1999) Religion and depression: a review of the literature by Twin Research 2: $126-136$

11. Colin Pritchard, S Amanullah (2007) An analysis of suicide and undetermined deats in 17 predominantly Islamic countries contrasted with the UK. Psychological Medicine 37: 421430.

12. Eskin M (2004) The effects of religious versus secular education on suicide ideation and suicidal attitudes in adolescents in Turkey. Social Psychiatry and Psychiatric Epidemiology 39(7).

13. Harold H.G Koenig and saad al shohaib (2014) Health and wellbeing in Islamic societies, background, research, applications, springer international publishing (suicide $\mathrm{p}$ 135).

14. Ahmed M. Abdel-Khalek, Ghada K. Eid (2011) Religiosity and its association with subjective well-being and depression among Kuwaiti and Palestinian Muslim children and adolescents, Mental Health, Religion \& Culture 14 (2): 117-127.

15. Habib Tiliouine, Robert A. Cummins, Melanie Davern (2009) Islamic religiosity, subjective well-being, and health, Mental Health, Religion \& Culture 12(1): 55-74.

16. Azhar MZ, Varma SL (1995a) Religious psychotherapy in depressive patients. Psychotherapy and Psychosomatics 63: $165-173$.

17. Azhar MZ, Varma SL (1995b) Religious psychotherapy as management of bereavement. Acta Psychiatrica Scandinavica 91: 233-235.

18. Razali SM, Hasanah CI, Aminah K, Subramaniam M (1998) Religious-sociocultural psychotherapy in patients with anxiety and depression. Australian and New Zealand Journal of Psychiatry 32: 867-872.

19. Harold H.G Koenig and Saad al shohaib Health and well being in Islamic societies, background, research, applications, springer international publishing ((Alcohol use/Abuse) p 155.

20. Harold H.G Koenig and saad al shohaib Health and wellbeing in Islamic societies, background, research, applications, springer international publishing ((Alcohol use/Abuse) p157.

21. https://aa-quebec.org/aaqc_wp/les-douze-etapes/

22. http://www.aa-francophonie.org/prieres.html

23. Ferri M, Amato L, Davoli M (2006) Alcoholics Anonymous and other 12-step programmes for alcohol dependence (Review) 3.

24. Harold G Koenig (2001) Religion and medicine ii: religion, mental health, and related behaviors int'l. $\mathrm{j}$. psychiatry in medicine 31(1): 97-109.

Copyright: (C2020 Benali Abdeslam. This is an open-access article distributed under the terms of the Creative Commons Attribution License, which permits unrestricted use, distribution, and reproduction in any medium, provided the original author and source are credited. 